\title{
Periodic Measurements of Passive Proton Beam Width Using Radiochromic Film in Fixed Gantry System
}

\author{
Tomohiro Shimozato ${ }^{1 *}$, Keisuke Yasui2 ${ }^{2}$, Hireto Kinou ${ }^{3}$, Fumiaki Komatsu ${ }^{4}$ \\ ${ }^{1}$ Faculty of Radiological Technology, School of Health Sciences, Gifu University of Medical Science, Seki, Japan \\ ${ }^{2}$ Faculty of Radiological Technology, School of Health Science, Fujita Health University, Toyoake, Japan \\ ${ }^{3}$ Nagoya Proton Therapy Center, Nagoya City West Medical Center, Nagoya, Japan \\ ${ }^{4}$ Department of Radiotherapy, Gifu University Hospital, Gifu, Japan \\ Email: *shimo-p@umin.ac.jp, k-yasui@fujita-hu.ac.jp, h.kinou.24@west-med.jp, f.komatsu5246@gmail.com
}

How to cite this paper: Shimozato, T., Yasui, K., Kinou, H. and Komatsu, F. (2019) Periodic Measurements of Passive Proton Beam Width Using Radiochromic Film in Fixed Gantry System. International Journal of Medical Physics, Clinical Engineering and Radiation Oncology, 8, 193-203.

https://doi.org/10.4236/ijmpcero.2019.84017

Received: August 13, 2019

Accepted: September 13, 2019

Published: September 16, 2019

Copyright (c) 2019 by author(s) and Scientific Research Publishing Inc. This work is licensed under the Creative Commons Attribution International License (CC BY 4.0).

http://creativecommons.org/licenses/by/4.0/

\begin{abstract}
Background and Aim: Irradiation methods such as double scattering method and spot scanning method have been used in proton beam treatment devices. In the scattering method, a ridge filter or a range modulation wheel is used to create a spread-out Bragg peak, but the distribution at the patient position may change due to positional deviation of the incident beam. Therefore, assessment of the incident position of the beam is very important even in the scattering method. To investigate the width and distribution of the proton beam before entering the RMW, a radiochromic film was installed at the outlet of the transport pipe and the entrance of the profile-monitoring detector. Methods. In this study, the distributions of the beam at the exit of the transport pipe and the entrance of the monitor detector were measured using films. The beam width was measured from the full width at half maximum of the profile obtained from the distribution. Measurements were conducted every month for 10 months. Results. Beams of widths ranging from 1.82 to $2.30 \mathrm{~mm}$ in the horizontal direction and 4.25 to $5.33 \mathrm{~mm}$ in the vertical direction were outputted from the exit of the transport pipe. Beams of widths ranging from 2.16 to $2.67 \mathrm{~mm}$ in the horizontal direction and 4.06 to 5.31 $\mathrm{mm}$ in the vertical direction were outputted from the entrance of the monitor detector. The maximum width fluctuation for 10 months was $0.55 \mathrm{~mm}$ in the horizontal direction and $1.26 \mathrm{~mm}$ in the vertical direction at the entrance of the monitor detector. Conclusions. The distribution was obtained before the proton beam was scattered by the scatterer, and then we propose a method to periodically measure and monitor the changes in the beam distributions every month.
\end{abstract}




\section{Keywords}

Proton Beam, Beam Width, Radiochromic Film, Periodic Measurement

\section{Introduction}

In external proton radiation therapy, irradiation of a patient's tumor is performed by methods such as double scattering method [1] [2] and spot scanning method [2] [3]. In dynamic beam delivery such as the spot scanning irradiation method, the accelerated thin pencil beam is controlled by the scanning electromagnet until it is irradiated on the patient, so changes in the shape and width of the beam may affect the dose administered to the patient. Therefore, such changes are controlled through regular measurements in the spot scanning method [4].

The passive irradiation method using a double scatterer widens the two-dimensional distribution of the beam before it reaches the patient position. In this method, highly accurate control of the beam position that is incident on the scatterer is not required because the scattered beam spreads before it hits the ridge filter. However, previous reports using Monte Carlo simulations have shown that the fluctuation of the beam before entering the range modulation wheel (RMW) affects the spread-out Bragg peak (SOBP) and penumbra at the patient position [5] [6]. If the RMW is located upstream of the scatterer or the RMW functions as the primary scatterer, it is expected that the change in position and shape of the beam will affect the width and depth of the SOBP at the patient position. The proton beam transmitted through the RMW is then expanded by the second scatterer to reach the patient position. As described above, depending on the entrance position to the RMW, there is a possibility that the width and depth of the SOBP at the patient position may be affected. Hence, it is important to assess the beam position and the shape outputted from the acceleration tube through periodic inspections.

In this study, we used the irradiation device at Nagoya Proton Therapy Center (NPTC) [7], which is equipped with an RMW that serves as the primary scatterer, as shown in Figure 1. This irradiation equipment is equipped with a profile monitoring detector, which monitors the position of the proton beam. By monitoring the beam position, this profile monitoring detector performs the function of turning off the beam when abnormal deviation is detected. However, it does not have a feedback function to the irradiation device in the case of small displacements; it is a detector in order to turning on the interlock thoroughly. In this way, in the current system, we can know the positional deviation of the proton beam only after the interlock is activated, but it is impossible to periodically check the displacement and shape of the proton beam. If we can obtain the beam position information beforehand, we can quickly detect defective components or operational problems in the equipment and deal with them before a 


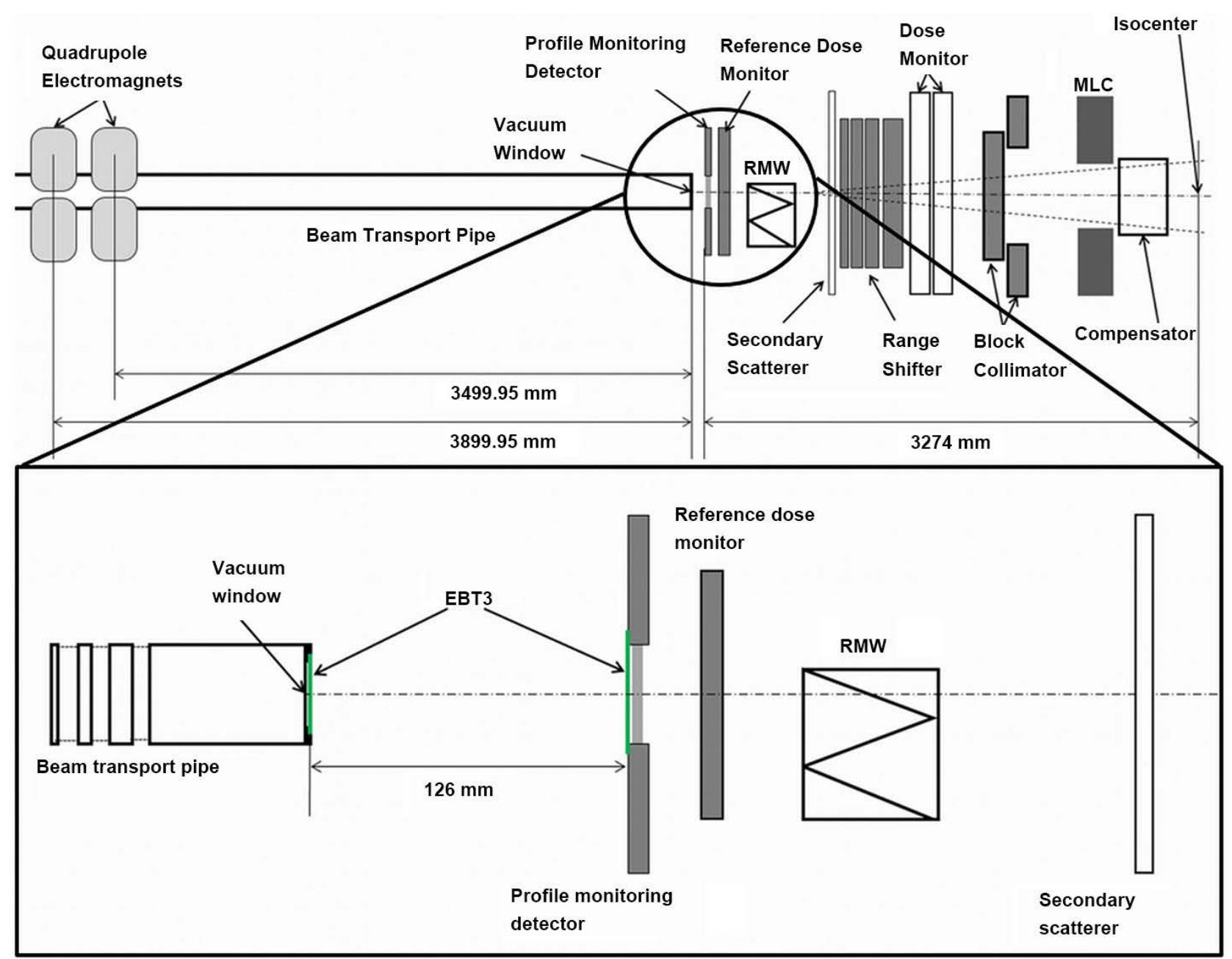

Figure 1. Schematic representations of the passive scattering nozzle of Hitachi PROBEAT III machine at the FX irradiation room. The figure was edited with reference to the figure described in reference 7.

major breakdown occurs. We attempted to measure with a Gafchromic film, as it is easily measurable at regular intervals. As we know, a Gafchromic film is easy to process; it can be processed in a room with normal light and the results can be obtained immediately after irradiation. To investigate the width and distribution of the proton beam before entering the RMW, a radiochromic film was installed at the outlet of the transport pipe and the entrance of the profile-monitoring detector. A simple distribution acquisition method was proposed based on this arrangement. Further, the change in the beam width was investigated by comparing the periodically measured values.

\section{Materials and Methods}

\subsection{Proton Beam Accelerator System}

The proton accelerator system PROBEAT III (Hitachi Ltd.) is an irregularly shaped synchrotron with a 7-MeV pre-linear accelerator. The extraction of protons from the synchrotron ring was performed using a slow extraction scheme. 
The accelerated protons are sent to the treatment rooms through a beam transport pipe. The NPTC has three treatment rooms: two rooms are equipped with isocentric gantries that rotate 360 around the patient and the third room is equipped with a fixed beamline in the horizontal direction. One gantry treatment room (G1) has a scanning treatment delivery nozzle. The other gantry treatment room (G2) and the fixed treatment room (FX) have passive scattering treatment delivery nozzles. The RMW inserted in the accelerator is equipped to form an SOBP, the width of which can be varied by gating the beam against the rotation angle of the wheel. A multi-leaf collimator (MLC) is placed in the passive irradiation system. Figure 1 shows a schematic of the passive scattering nozzle. The narrow proton beam entering the nozzle is spread laterally by a double scattering system. The RMW that produces a uniform SOBP acts as the first scatterer. The secondary scatterer is located downstream of the RMW. The profile monitor in front of the RMW is a detector for activating the interlock when the beam position changes significantly. This monitor has not been subjected to feedback from the beam control.

Eight beams of varying energies were extracted from the accelerator for the passive irradiation system: 250, 225, 200, 180, 160, 140, 120, and $100 \mathrm{MeV}$. Five such beams $(225,200,180,160$, and $140 \mathrm{MeV})$ could be selected in the FX treatment room, where only the $14-\mathrm{cm}$-diameter field was available and there were two wheels and one secondary scatterer. One wheel corresponded to one beam energy in each uncollimated field size. The rotating RMW modulates the range and weight of multiple Bragg peaks to produce a uniform SOBP. The RMWs rotate at $400 \mathrm{rpm}$ and produce six range modulations per revolution. In this study, all measurements were performed before the proton beam was incident on the RMW because of which the operation of the RMW was not affected.

\subsection{Measurement of Beam Distribution Using Radiochromic Film}

Proton beams of 225, 200,180,160, and $140 \mathrm{MeV}$ were used in the FX treatment room equipped with the proton beam accelerator system at the NPTC. The proton beam distribution for each value of beam energy outside the vacuum window of the beam transport pipe was measured using a radiochromic film (Gafchromic film EBT3; Lot\#: 06051040; Ashland Inc., Bridgewater, NJ). The beam distributions at the entrance of the profile-monitoring detector were also measured. In this study, the exit of the vacuum window was defined as upstream, and the entrance of the profile-monitoring detector was defined as downstream. The irradiation dose was set at $0.5 \mathrm{MU}$. We defined the monitor unit (MU) by using the reference condition: 1-Gy dose delivered to the center of 1-L volume (field size of $10 \times 10 \mathrm{~cm}^{2}$ determined by the MLC and SOBP width of $10 \mathrm{~g} / \mathrm{cm}^{2}$ ) of water at the isocenter, with the most distal point in the volume at $21.8 \mathrm{~g} / \mathrm{cm}^{2}$ depth. Dose of $100 \mathrm{MU}$ was delivered by using an RMW for the $200 \mathrm{MeV}$ beam and 14-cm-diameter field. The profile-monitoring detector performs measurements to achieve interlock control when the position of the beam deviates from the 
prescribed position. This monitoring detector does not perform feedback control of the proton beam incident position. Therefore, it is considered that the distribution shape of the beam does not change with change in the dose.

The radiochromic film was fixed with tape to the outlet of the vacuum window on the upstream side and to the incident side of the profile-monitoring detector on the downstream side. The EBT3 film was installed such that the landscape direction of the film coincided with the horizontal direction and the frontal side was on the side where the beam was incident. Digitizing of the film density by the flatbed scanner was performed after letting its tabilize for 3 days after proton beam irradiation. Reading of the EBT3 film by the scanner was performed so that the landscape direction of the film was perpendicular to the operational direction of the scanner. The net optical density (OD) of the EBT3 film was obtained using a flatbed scanner (Offirio ES-10000G; Epson Seiko Co., Nagano, Japan), which was controlled by film scan software (FilmScan Version 2.8.15; PTW Freiburg, Germany) and digitized by film analysis software (Film Analyze Version 1.8.2; PTW Freiburg, Germany). Reading of the EBT3 film was performed taking into account the characteristics of the film and scanner reported in a previous study [8]. Lateral profile curves obtained from two-dimensional data were used to acquire the full width at half maximum (FWHM). In this study, the proton beam width was defined as the FWHM. The horizontal direction was defined as the $\mathrm{X}$-axis, and the vertical direction was defined as the Y-axis. The films were irradiated every month. The beam widths (FWHM) obtained from the profile curves were compared for the X-and Y-axes.

The measurement period was 10 months (from March to December 2017). The reproducibility and rate of change of the beam width (FWHM) for each lateral profile curve were investigated using this 10-month data.

\section{Results}

As an example, images of the EBT3 film irradiated with the $180-\mathrm{MeV}$ proton beam in the upstream and downstream directions in the film installation position shown in Figure 1 are depicted in Figure 2(a) and Figure 2(b). It was observed that the two-dimensional distribution of the beam is not a perfect ellipse but is an irregular shape. Similar results were obtained for other energy distributions.

The upstream and downstream two-dimensional distributions for each proton energy value are shown in Figure 3 for the same period. Figures 3(a)-(e) show the upstream two-dimensional distributions for each proton energy value and Figures 3(f)-(j) show the corresponding downstream two-dimensional distributions. The two-dimensional distributions are different in shape depending on the value of the beam energy. In addition, the upstream distribution is more irregular than the downstream distribution. 


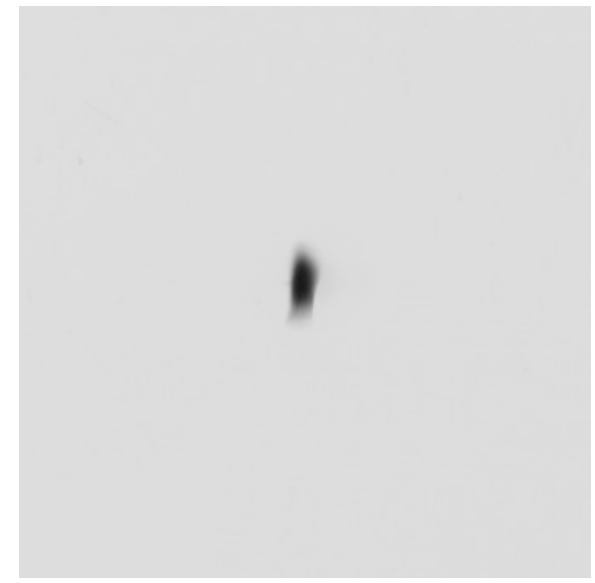

(a)

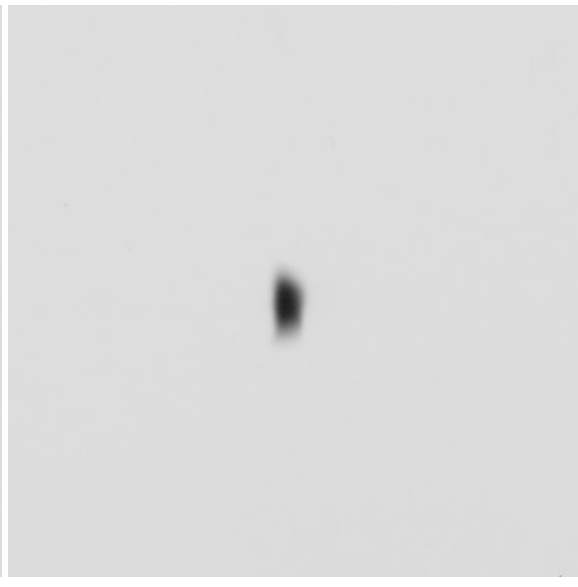

(b)

Figure 2. The measured fluence distributions for the $180-\mathrm{MeV}$ proton beam. (a) Upstream; (b) Downstream.

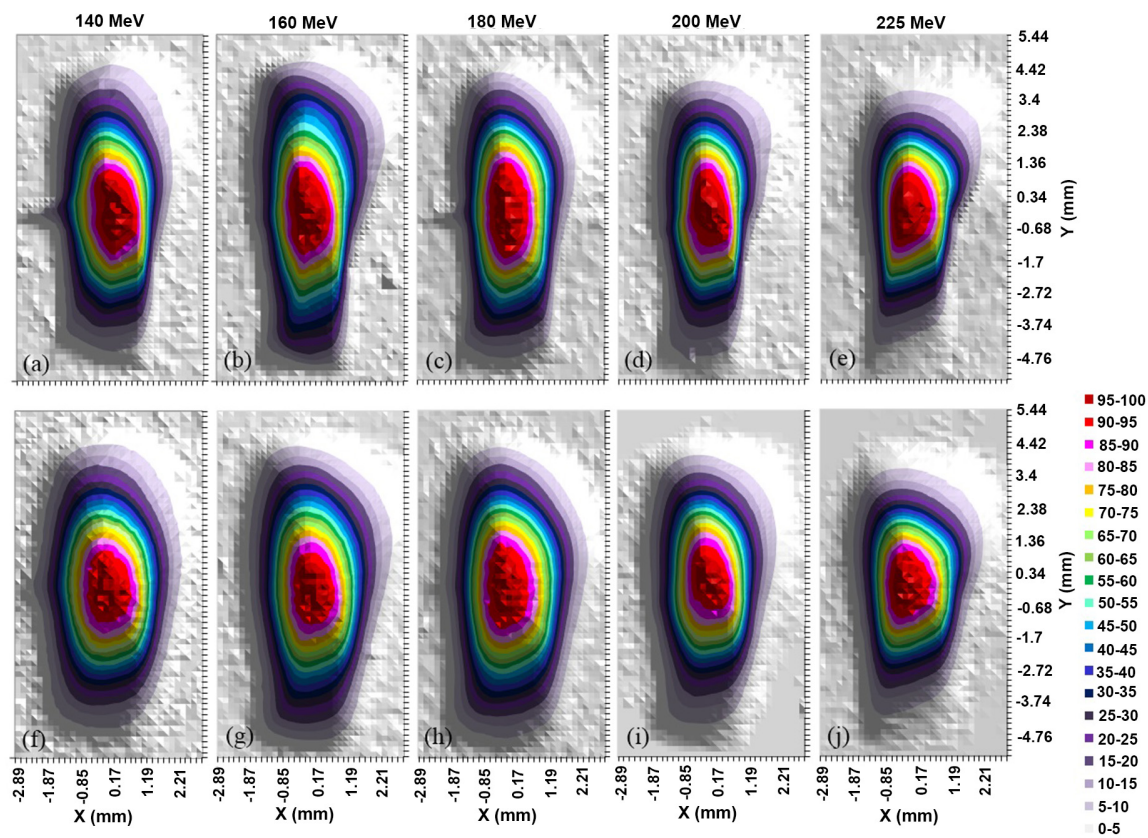

Figure 3. The two-dimensional distributions by film measurement in air during the same period for each proton energy value. (a)-(e) The distributions at the exit of the beam transport pipe (downstream); (f)-(j) The distributions at the entrance of the profile monitor detector (upstream).

Figure 4 shows the two-dimensional upstream distributions for a $180-\mathrm{MeV}$ proton beam for only 10 months due to the reason for using the facility. The changes in the upstream beam shape are the same as those in the downstream, so the other energy shape changes are considered from the downstream shapes shown in Figures 5-7. The two-dimensional distributions indicated no significant change in 10 months. The two-dimensional downstream distributions across 10 months for 140, 180, and $225 \mathrm{MeV}$ proton beams are shown from Figures 5-7, respectively. The shapes of the two-dimensional downstream 


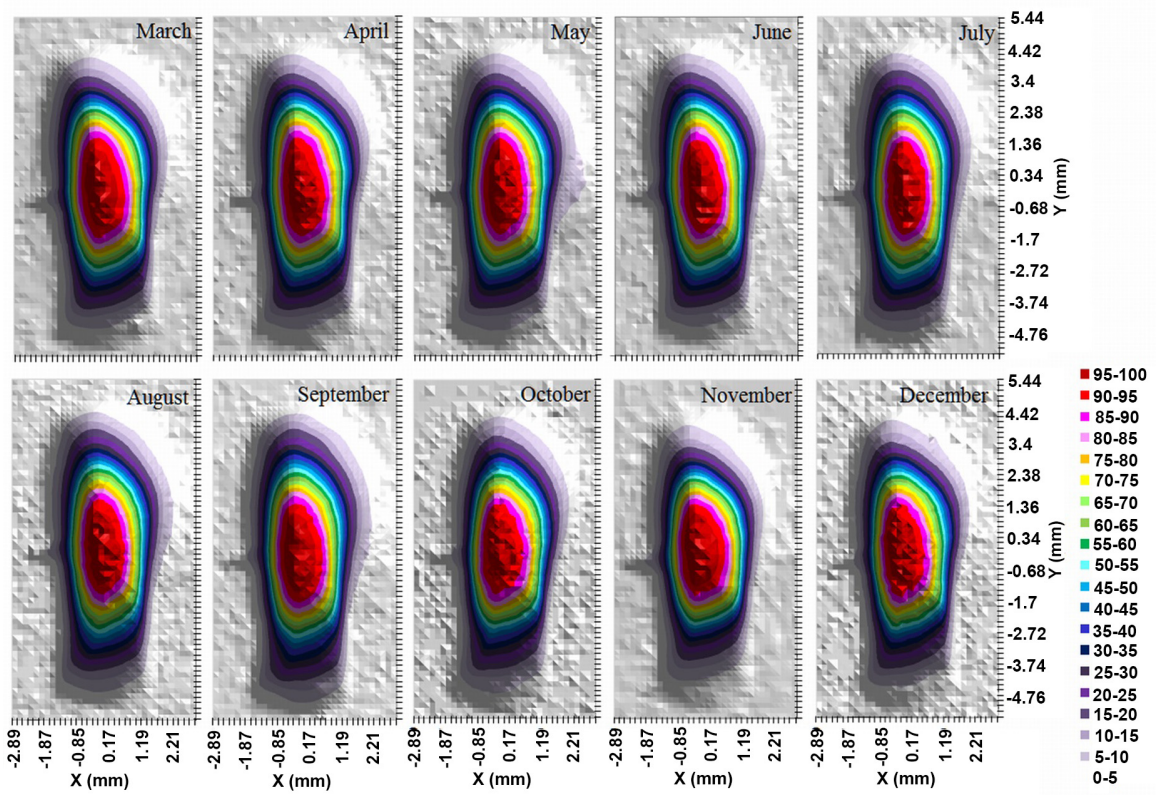

Figure 4. The two-dimensional upstream distributions across 10 months for the $180-\mathrm{MeV}$ proton beam.

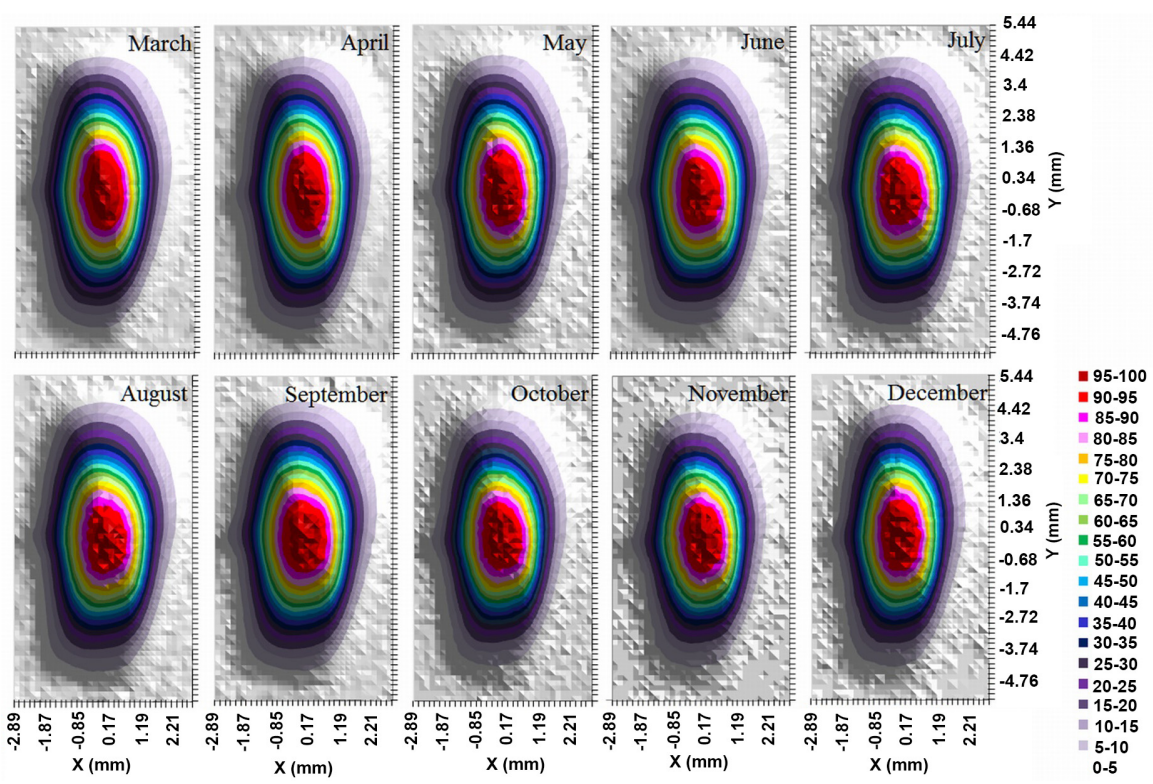

Figure 5. The two-dimensional downstream distributions across 10 months for the $140-\mathrm{MeV}$ proton beam.

Distributions were not significantly changed with regard to their energies over the measured 10 months.

Figure 8 shows the up stream and downstream mean beam widths and twice the standard deviation (2SD) of the FWHM of the X-axis profile and Y-axis profile for each proton energy value. As a general trend, there is almost no change in the beam width due to proton energy along the $\mathrm{X}$-axis, whereas the beam width along the $\mathrm{Y}$-axis becomes smaller as the proton energy becomes higher. In the measurement taken over 10 months, the change in the proton beam width 

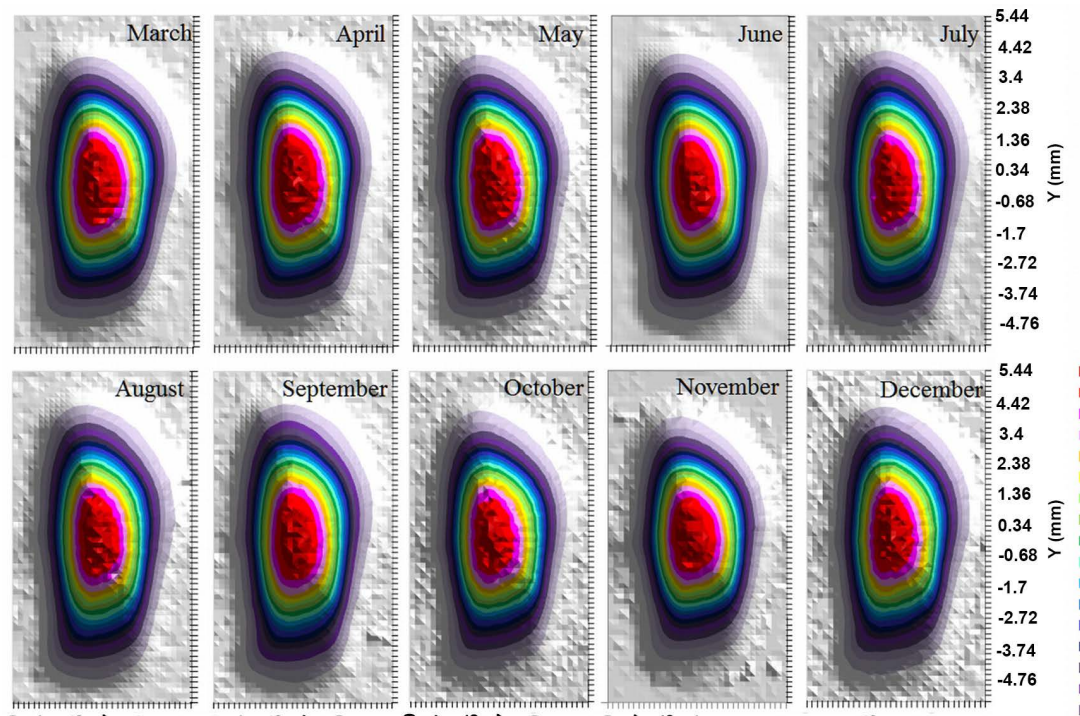

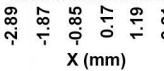

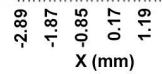

离

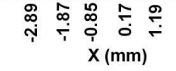

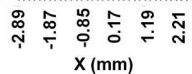

$-95-100$
$=90-95$

90-95

85-90

$80-85$
$75-80$
$70-75$

$70-75$

$60-65$

$55-60$

$\begin{array}{r}50-55 \\ -45-50 \\ \hline\end{array}$

$40-45$

$35-40$

- $30-35$

- $30-35$
- $25-30$

$-25-30$
$-20-25$
$-15-20$

$15-20$
$10-15$

$10-15$
$-5-10$

Figure 6. The two-dimensional downstream distributions across 10 months for the $180-\mathrm{MeV}$ proton beam.
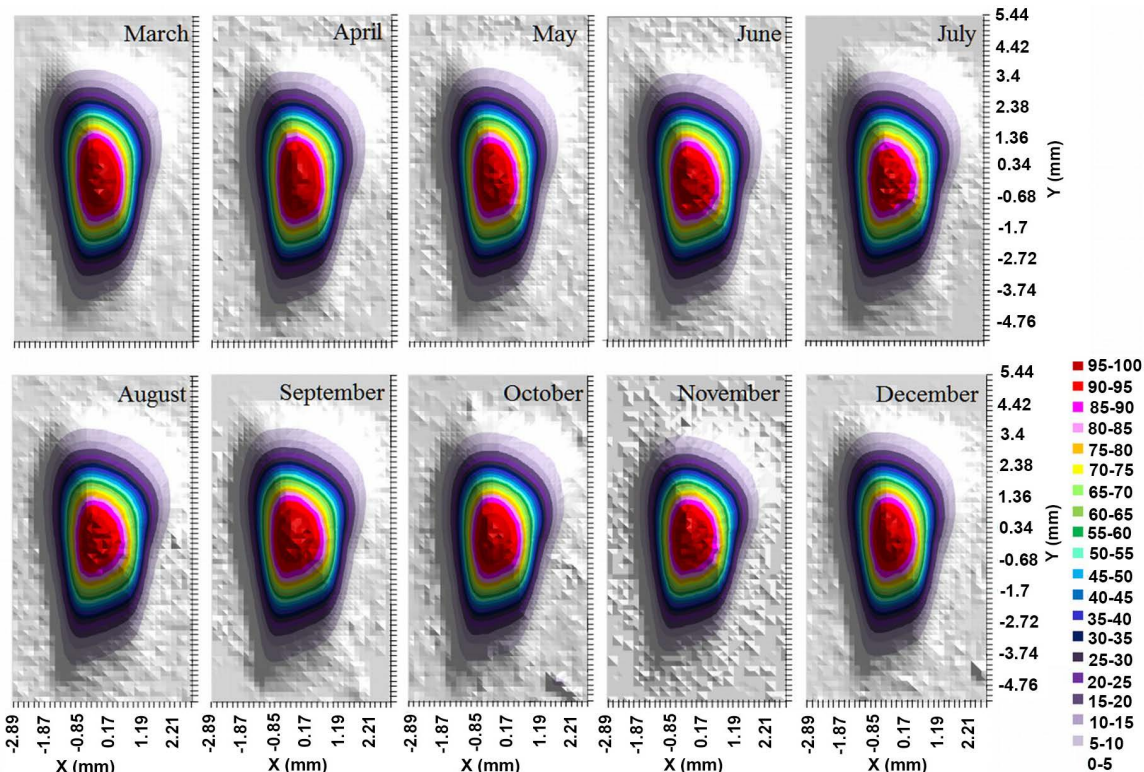

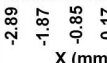

Figure 7. The two-dimensional downstream distributions across 10 months for the $225-\mathrm{MeV}$ proton beam.

was larger along the $\mathrm{Y}$-axis than along the $\mathrm{X}$-axis. Further, it was unrelated to the fluctuations in the beam width due to proton energy difference. The mean upstream beam widths obtained from the profile curves were between $4.25 \pm 0.25$ $\mathrm{mm}$ (mean $\pm 2 \mathrm{SD}$ ) for $225 \mathrm{MeV}$ to $5.33 \pm 0.46 \mathrm{~mm}$ for $160 \mathrm{MeV}$ along the $\mathrm{Y}$-axis and between $1.82 \pm 0.15 \mathrm{~mm}$ for $200 \mathrm{MeV}$ to $2.30 \pm 0.09 \mathrm{~mm}$ for $180 \mathrm{MeV}$ along the $\mathrm{X}$-axis. The mean downstream beam widths were between $4.06 \pm 0.27 \mathrm{~mm}$ for $225 \mathrm{MeV}$ to $5.31 \pm 0.71 \mathrm{~mm}$ for $160 \mathrm{MeV}$ along the $\mathrm{Y}$-axis and between 2.16 $\pm 0.17 \mathrm{~mm}$ for $200 \mathrm{MeV}$ to $2.67 \pm 0.10 \mathrm{~mm}$ for $180 \mathrm{MeV}$ along the $\mathrm{X}$-axis. Table 1 


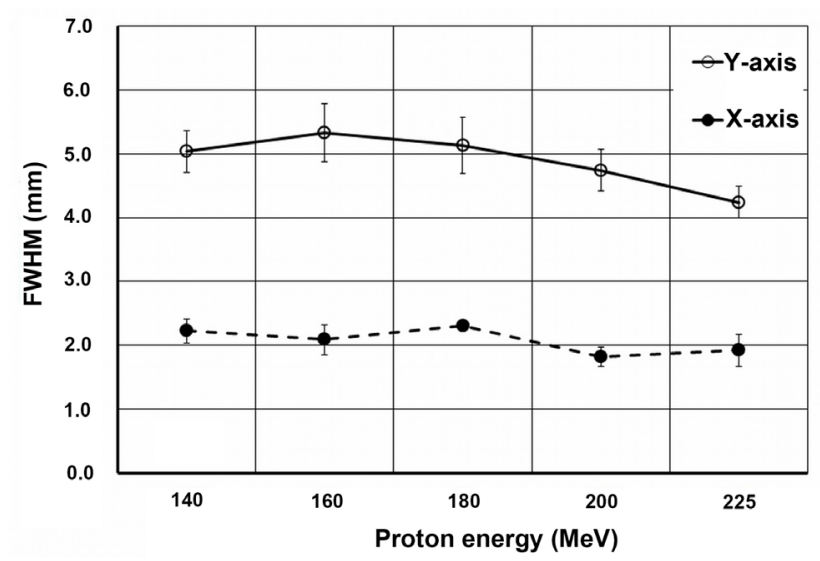

(a)

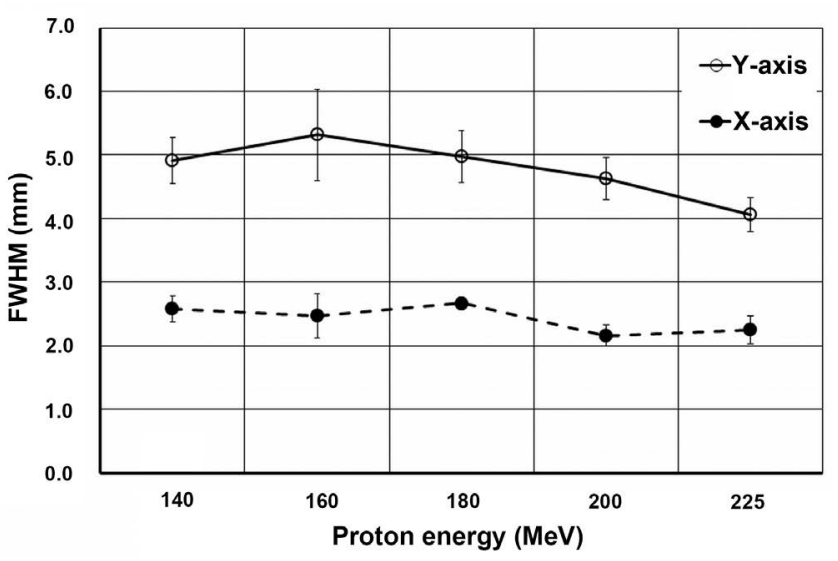

(b)

Figure 8. (a) The upstream proton beam width (FWHM) of the lateral profiles in air for each proton energy value; (b) The downstream proton beam width (FWHM) of the lateral profiles in air for each proton energy value.

Table 1. The mean values, 2SD, and the maximum fluctuation range of the beam width (FWHM).

\begin{tabular}{|c|c|c|c|c|c|}
\hline \multirow{2}{*}{ Position } & \multirow{2}{*}{ Direction } & \multirow{2}{*}{ Proton energy } & Mean & $2 \mathrm{SD}$ & Maximum range \\
\hline & & & $(\mathrm{mm})$ & $(\mathrm{mm})$ & $(\mathrm{mm})$ \\
\hline \multirow[t]{10}{*}{ Upstream } & $\mathrm{X}$-axis & $140 \mathrm{MeV}$ & 2.23 & 0.19 & 0.29 \\
\hline & & $160 \mathrm{MeV}$ & 2.09 & 0.23 & 0.35 \\
\hline & & $180 \mathrm{MeV}$ & 2.30 & 0.09 & 0.15 \\
\hline & & $200 \mathrm{MeV}$ & 1.82 & 0.15 & 0.22 \\
\hline & & $225 \mathrm{MeV}$ & 1.92 & 0.25 & 0.37 \\
\hline & Y-axis & $140 \mathrm{MeV}$ & 5.04 & 0.33 & 0.54 \\
\hline & & $160 \mathrm{MeV}$ & 5.33 & 0.46 & 0.71 \\
\hline & & $180 \mathrm{MeV}$ & 5.14 & 0.45 & 0.70 \\
\hline & & $200 \mathrm{MeV}$ & 4.75 & 0.33 & 0.56 \\
\hline & & $225 \mathrm{MeV}$ & 4.25 & 0.25 & 0.45 \\
\hline \multirow[t]{10}{*}{ Downstream } & $\mathrm{X}$-axis & $140 \mathrm{MeV}$ & 2.58 & 0.20 & 0.30 \\
\hline & & $160 \mathrm{MeV}$ & 2.46 & 0.34 & 0.55 \\
\hline & & $180 \mathrm{MeV}$ & 2.67 & 0.10 & 0.18 \\
\hline & & $200 \mathrm{MeV}$ & 2.16 & 0.17 & 0.25 \\
\hline & & $225 \mathrm{MeV}$ & 2.25 & 0.22 & 0.33 \\
\hline & Y-axis & $140 \mathrm{MeV}$ & 4.91 & 0.37 & 0.54 \\
\hline & & $160 \mathrm{MeV}$ & 5.31 & 0.71 & 1.26 \\
\hline & & $180 \mathrm{MeV}$ & 4.98 & 0.41 & 0.66 \\
\hline & & $200 \mathrm{MeV}$ & 4.63 & 0.33 & 0.49 \\
\hline & & $225 \mathrm{MeV}$ & 4.06 & 0.27 & 0.46 \\
\hline
\end{tabular}

shows the mean value, 2SD, and the maximum fluctuation range of the beam width (FWHM).

\section{Discussions}

When compared with the upstream distribution, the distribution at the down- 
stream appears to be of an irregular shape approaching an ellipse due to the influence of scattering by air. The reason for the difference in shape is considered to be the fact that the focusing of the beam is adjusted by the quadrupole electromagnets at the position where it is incident on the RMW.

It is considered that the difference in widths along the $\mathrm{X}$-axis and $\mathrm{Y}$-axis is influenced by the difference in the individual magnetic field strengths of the quadrupole electromagnet used for beam focusing. The role of the quadrupole electromagnet is to collect proton particles, which mainly deviate from the center of the transport pipe, to the center. The plurality of the electromagnets is installed on a synchrotron or a transport pipe, and the shape of the beam is the result of the control exerted by these electromagnets. A comparison of the values measured across 10 months shows almost no change in the distribution shape and the beam width. The maximum width fluctuation for 10 months was $0.55 \mathrm{~mm}$ in the horizontal direction and $1.26 \mathrm{~mm}$ in the vertical direction at the entrance of the profile monitor detector. Although the irradiation was of low MU, extremely stable irradiation could be performed.

The changes in the beam widths observed during the periodic measurements are considered to be due to contraction and expansion of the building because of influences of temperature, atmospheric pressure, humidity, and the like. In addition, changes in the ground are considered as long-term changes, and there may be influence of the subsidence and fluctuation of the ground. The increase and decrease in temperature were considered during the measurement period; however, fluctuations in the beam width due to temperature have not been clarified in this study. Beam width measurements were performed in this study, but the effects of these changes on the output of the proton beam is a subject for future study.

\section{Conclusion}

This study presented a practical method to measure the width and two-dimensional distribution of the proton beam incident on the profile monitor. The profile monitor detector of the proton beam therapy system used in this study is not equipped with a feedback mechanism and it is considered useful in obtaining measurements such as that of the beam width simply by periodic inspection. It was confirmed that the beam width and shape of the two-dimensional distribution are different depending on the difference in energy. In addition, the possibility of continuous measurement suggests its application to periodic inspections. The reason for the change in the shape of the proton beam could not be clarified in this paper, but it is expected to be resolved with continuing investigation. This method was evaluated with a very low proton dose; in reality, the treatment is performed with a dose that is approximately 100 times the dose considered here. It is necessary to use a less sensitive film to evaluate the proposed method with the actual dose, which will be considered in a future task.

\section{Financial Disclosure}

There are no funding sources or affiliations to disclose. 


\section{Conflicts of Interest}

The authors report no conflict of interest.

\section{References}

[1] De Laney, T.F. and Kooy, H.M. (2008) Proton and Charged Particle Radiotherapy. Lippincott Williams \& Wilkins, Philadelphia, 33-49.

[2] ICRU (2007) Prescribing, Recording, and Reporting Proton-Beam Therapy. ICRU Report No. 78. International Commission on Radiation Units and Measurements, Bethesda.

[3] Kanai, T., Kawachi, K., Kumamoto, Y., et al. (1980) Spot Scanning System for Proton Radiotherapy. Medical Physics, 7, 365-369. https://doi.org/10.1118/1.594693

[4] Titt, U., Mirkovic, D., Sawakuchi, G.O., et al. (2010) Adjustment of the Lateral and Longitudinal Size of Scanned Proton Beam Spots Using a Pre-Absorber to Optimize Penumbrae and Delivery Efficiency. Physics in Medicine and Biology, 55, 7097-7106. https://doi.org/10.1088/0031-9155/55/23/S10

[5] Polf, J.C., Harvey, M.C., Titt, U., Newhauser, W.D. and Smith, A.R. (2007) Initial Beam Size Study for Passive Scatter Proton Therapy. I. Monte Carlo Verification. Medical Physics, 34, 4213-4218. https://doi.org/10.1118/1.2767934

[6] Polf, J.C., Harvey, M.C. and Smith, A.R. (2007) Initial Beam Size Study for Passive Scatter Proton Therapy. II. Changes in Delivered Depth Dose Profiles. Medical Physics, 34, 4219-4222. https://doi.org/10.1118/1.2789497

[7] Toshito, T., Omachi, C., Kibe, Y., et al. (2016) A Proton Therapy System in Nagoya Proton Therapy Center. Australasian Physical and Engineering Sciences in Medicine, 39, 645-654. https://doi.org/10.1007/s13246-016-0456-8

[8] Ferreira, B.C., Lopes, M.C. and Capela, M. (2009) Evaluation of an Epson Flatbed Scanner to Read Gafchromic EBT Films for Radiation Dosimetry. Physics in Medicine and Biology, 54, 1073-1085. https://doi.org/10.1088/0031-9155/54/4/017 